\title{
The quantum game
}

\author{
With the launch of the Quantum Technologies Flagship, the European Union is looking to become a \\ major player in the upcoming quantum revolution, reaping benefits both for technology development \\ and wealth creation for the European society.
}

Following the publication of the Quantum Manifesto (http://qurope.eu/manifesto), a $€ 1$ billion flagship project was announced in May 2016, combining European and national funding to promote quantum technologies (QT) over the next decade ${ }^{1}$. An independent high-level steering committee of academics and industry experts compiled a strategic research agenda (a long-term roadmap), which was handed over in February 2017 to Khalil Rouhana, Deputy Director-General of the European Commission (EC) department for Communications Networks, Content and Technology ${ }^{2}$, at an event organized by the Maltese government (currently presiding over the European Council). The next step in the ramp-up phase will be determining the governance structure and submitting the final strategic proposal in September 2017. The first call for proposals will be rolled out this autumn with funded projects scheduled to start in January 2019.

The primary aim of the QT Flagship will be delivering new technology based on quantum physics in four main areas: communication, simulation, sensing and metrology, and computation. These pillars are intended to improve both the technology and the education landscape, not only delivering QT-based commercial products (hardware/software), but also producing wealth for society, reshaping the workforce (who will either participate in the production of QT devices or use them in their jobs) and transforming the market itself. By playing a leading role in the industry springing from the upcoming quantum revolution, Europe hopes to become an attractive region for innovation, investment, and native and global talent.

Undoubtedly, Europe has a strong academic presence in the field, but a translation in technology is not straight forward, and competition is fierce: China, Japan, Russia, Singapore and Australia have each launched their own national research programs, while tech giants like Microsoft, Google and $\mathrm{IBM}^{3}$ are also investing heavily. Unlike companies, which strategically target the delivery of a final product ${ }^{4}$, the EC faces the more difficult challenge of becoming technologically competitive while preserving the principles of openness, fairness and transparency in the selection of projects and distribution of funding. This certainly sets a different tone for the whole endeavour.

Concerns over the fair but fast distribution of funding, as well as ways to implement an impartial peer-review process with fundamental and industrial aspects, were repeatedly brought up during the open discussions in Malta. The involvement of industry is crucial to the success of the Flagship, and actions to de-risk early investments need to be put into place. Simplifying intellectual property legislation across Europe in order to speed up the protection process and avoid fragmentation in different countries is also a necessity. A rigorous way to assess the technology that comes out through validation platforms must also be established. Overall, a clear strategic direction must be implemented to ensure the strong link between all projects and maximize impact.

Finally, there is the bigger issue of balancing inclusivity (embracing all member countries independent of their contributions through national funding initiatives) with excellence (promoting the best research across the continent). It is indeed clear that some European countries are in a more advanced position in the race for the deployment of quantum technologies. A number of member states, including Hungary and Austria, have already announced national initiatives (€11 million and $€ 32$ million, respectively, over the next 5-6 years), and others will follow (Germany, for example). The heftiest national programme is undoubtedly the UK's quantum hubs initiative (http://uknqt.epsrc.ac.uk), which pledges $£ 350$ million for the first five years. Although several countries have not planned dedicated budgets yet, they can offer different skills: Malta, for example, may well prove to be a very useful test-bed for rolling out quantum networks. With QT considered an essential upcoming industry by the EC, all countries should reassess their strengths and find ways to participate in the Flagship.

The EC on the other side is sending a clear message that this Flagship will not constitute business as usual. The available funding is not meant to support quantum research but rather promote an ambitious agenda of delivering disruptive technologies. While product commercialization is also

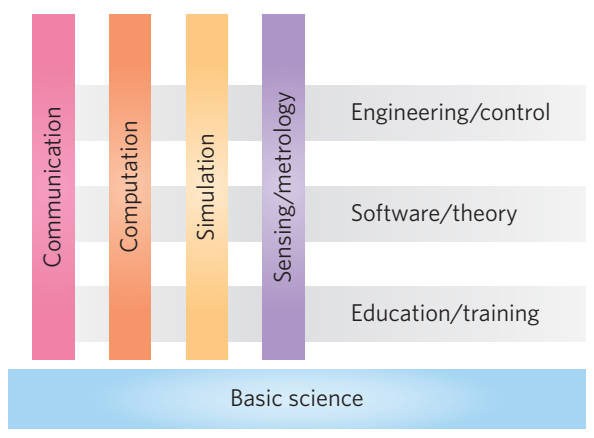

The outline of the strategic research agenda of the QT Flagship. Reproduced from ref. 5, EC.

the main goal for the Graphene Flagship, identifying those areas where graphene is more likely to be a game-changer is part of the journey. In contrast, the QT Flagship has already decided which applications will be pursued, with the material strategies being more tightly constrained in more developed pillars: for example, advanced platforms such as trapped ions and superconducting qubits will be mainly adopted to realize a quantum computer, whereas a broader range of approaches will be investigated simultaneously in the quantum simulations pillar. Although less widely studied platforms are not entirely disregarded, it has become clear that new ideas will only be integrated if shown to be commercially promising.

In addressing these issues, the EC has promised a light-and-lean style of governance with strong links to the EC and the QT community, combining the flexibility of a decentralized programme with the coordination of focused initiatives, without compromising its highly mission-oriented character. It remains to be seen if such an approach will make Europe a major player on the quantum scene.

\footnotetext{
References

1. European Commission will launch $€ 1$ billion quantum technologies flagship. European Commission (17 May 2016); http://go.nature.com/2mpP4OA

2. Rouhana, K. The Quantum Technology Flagship takes shape in Malta. European Commission (22 February 2017); http://go.nature.com/2mnBM3G

3. Castelvecchi, D. Nature 543, 159 (2017).

4. Mohseni, M. et al. Nature 543, 171-174 (2017).

5. Quantum Technologies Flagship Intermediate Report (European Commission, 2017); http://go.nature.com/2m48x41
} 